\title{
Proof of the planar double bubble conjecture using metacalibration methods
}

\author{
Rebecca Dorff, Gary Lawlor, Donald Sampson and Brandon Wilson
}

(Communicated by Frank Morgan)

\begin{abstract}
We prove the double bubble conjecture in $\mathbb{R}^{2}$ : that the standard double bubble in $\mathbb{R}^{2}$ is boundary length-minimizing among all figures that separately enclose the same areas. Our independent proof is given using the new method of metacalibration, a generalization of traditional calibration methods useful in minimization problems with fixed volume constraints.
\end{abstract}

\section{Introduction}

Isoperimetric problems have had a long history. The earliest proofs that the circle maximizes area for a figure of given perimeter can be traced to the ancient Greeks. The first results are attributed to the second century mathematician Zenodorus. After more than a millennium, Steiner was the first to realize that the ancient Greek proofs were insufficient by modern standards and worked to complete them. Weierstrass, however, was the first to give a rigorous proof of the isoperimetric inequality, and furthered the development of analysis and calculus in order to do so [Siegel 2003].

While many different proofs exist for the isoperimetric inequality in two dimensions, few of these methods can be applied to generalizations of the problem, including having multiple enclosed areas or higher dimensional analogs. The traditional approach, and so far most successful, has been to use the calculus of variations to isolate properties of the boundary-minimizing figure and compare all possible figures of this type. Some advancements in "multiple bubble" problems were made this way by Frederick Almgren [1976], Jean Taylor [1976], and Frank Morgan [1994], who proved regularity results in $\mathbb{R}^{n}$ for $n \geq 4, n=3$, and $n=2$, respectively. Morgan realized that a careful analysis of minimizers in the plane was absent from the literature, and providing this, showed that perimeter-minimizing planar figures must consist of circular arcs meeting at vertices of degree three,

MSC2000: 49Q05, 49Q10, 53A10.

Keywords: calibration, metacalibration, double bubble, isoperimetric, optimization. 
forming 120-degree angles. This reduced the argument to listing all the combinatorial types meeting these requirements, possibly subject to some bounds on the number of components that each area may be broken up into. Using this result, students of the 1990 SMALL group under Frank Morgan proved that the standard double bubble was perimeter-minimizing among all figures separately enclosing two fixed areas [Foisy et al. 1993]. This method was also employed by Wacharin Wichiramala, whose doctoral dissertation proves the corresponding result for three separated areas. Unfortunately, this approach is marked by an ever-increasing combinatorial complexity. For example, Wichiramala's dissertation [2004] considered some fifty-four possible configurations in order to prove minimization of the standard triple bubble. This complexity proves to be a significant barrier to further results.

We present new proofs of the isoperimetric inequality in the plane, for one and two areas, using metacalibration, a new method of proof developed by Gary Lawlor. Metacalibration is an extension of previous work in the field of calibration that has been modified to handle a new class of minimization problems. Section 2 discusses metacalibration in further detail. A reformulation of ideas by Gromov [Milman and Schechtman 1986], Lawlor's proof of the two-dimensional isoperimetric inequality is given in Section 3. Lawlor also showed using metacalibration that the standard double bubble is perimeter-minimizing among all figures enclosing two equal areas. Section 4 contains the authors' generalization of this proof to any two fixed areas. Metacalibration has a strong potential for other applications in which the standard variational approach fails. Further work is currently being made in extending our results to other multiple bubble problems, including the as-yet-unproven triple bubble conjecture in $\mathbb{R}^{3}$.

\section{Metacalibration}

Each minimization problem ${ }^{1}$ can be expressed in the following terms. Let a set of constraints $C$ be given. Let $S$ be the set of all competitors $\sigma$ that satisfy $C$. For each competitor $\sigma \in S$, we define the function $P(\sigma)$ which expresses the quantity to be minimized (perimeter, energy, etc.). The minimizing property of a conjectured minimizer $\mu \in S$ is shown by proving $P(\mu) \leq P(\sigma)$ for all $\sigma \in S$.

Metacalibration solves minimization problems by comparing $P(\sigma)$ to an intermediary function $G(\sigma)$ which simplifies the conditions for comparison. This function is called a calibration function and is defined as follows.

\footnotetext{
${ }^{1}$ As the methods of metacalibration work identically for minimization as well as maximization problems, we will present these methods in the context of a minimization problem. Maximization problems are solved identically with the obvious inequalities reversed.
} 
Definition 2.1. A function $G$ calibrates a conjectured minimizer, $\mu \in S$, if for every competitor $\sigma \in S$,

(1) $P(\mu)=G(\mu)$,

(2) $G(\mu) \leq G(\sigma)$, and

(3) $G(\sigma) \leq P(\sigma)$.

The following theorem encapsulates this idea.

Theorem 2.2. Calibration Theorem. If a function $G$ calibrates a conjectured minimizer $\mu$ with respect to $P$, then $\mu$ minimizes the function $P$.

Proof. Take any competitor $\sigma \in S$. By the definition of a calibration, $P(\mu)=$ $G(\mu) \leq G(\sigma) \leq P(\sigma)$. Thus for any $\sigma \in S, P(\mu) \leq P(\sigma)$ and $\mu$ minimizes $P$.

One can see from the above arguments that this method yields simple and elegant results if we are able to identify a suitable calibration function $G(\sigma)$. Finding such a function is one of the difficulties of this method. Following is a short description of some characteristics of calibration functions.

In order to establish the necessary identities, calibration functions typically have the form of an integral. For example, if we parametrize $\sigma$ by some variable $t$, the necessary relations may be established by comparing the rate of change of $G(\sigma)$ and $P(\sigma)$ with respect to $t$. Suppose we find a function $d f_{\sigma} / d t=f_{\sigma}^{\prime}$ such that $f_{\sigma}^{\prime}(t) \leq P_{\sigma}^{\prime}(t)$, with equality on the minimizer. Letting $\sigma$ be parametrized by $t \in\left[t_{0}, t_{1}\right]$, we define the function $G(\sigma)=\int_{t_{o}}^{t_{1}} f_{\sigma}^{\prime} d t$. We find that $G(\sigma)$ will be a calibration function if $f_{\sigma}\left(t_{1}\right)-f_{\sigma}\left(t_{0}\right)$ is constant for all competitors, or at least minimal on $\mu$. If this is the case, $f_{\sigma}^{\prime}$ is called a calibration. In many cases these conditions allow us to explicitly determine the function $f_{\sigma}^{\prime}$. In any case however, solving these conditions will often give insight into the form or character of the necessary function $f_{\sigma}^{\prime}$.

In the past, calibrations have been functions of spacial variables, such as position or tangent vectors. In metacalibration, we also allow the calibration function $G(\sigma)$ to depend on characteristics of $\sigma$ itself. These may include variables such as arc length or enclosed area. Another useful tool of metacalibration is that it allows other variables to be defined abstractly, such as characteristics of the competitor under mapping or projection. These additional allowances of metacalibration allow calibration functions to consider a wider range of competitors, enabling metacalibration to take on various problems beyond the reach of standard methods.

In the following sections we show how two classical geometric optimization problems may be solved using the methods of metacalibration. While each is a previously solved problem, they demonstrate the usefulness of this method and its future extension into yet unsolved problems. 


\section{The circle is perimeter-minimizing in $\mathbb{R}^{2}$}

We begin our application of metacalibration with the classic example of the isoperimetric inequality in $\mathbb{R}^{2}$. Of all figures that enclose the same area in the plane, we wish to show that the circle of that area minimizes total perimeter. The minimizing property of the circle is shown using the calibration theorem of the previous section. To do this, we first define the function $G(\sigma)$, and subsequently prove that it satisfies each of the conditions for calibration.

Take an arbitrary competing figure $\sigma$ in $S$, the set of competitor figures $\left(C^{1}\right.$ manifolds) that enclose a fixed area $A_{0}$. We assume that the boundary of the figure is equal to the boundary of its interior. This ensures that $\sigma$ will have no obviously unnecessary perimeter elements that do not enclose area. Let an arbitrary axis for the parameter $t$ be given. We will parametrize $\sigma$ by a set of slicing lines perpendicular to the $t$-axis, whose position is determined by the variable $t$. In our depictions, this is an upward sweeping line with a vertical $t$-axis. We let $t_{0}$ be the bottom of the figure and $t_{1}$ the top, so that $\sigma$ is fully contained in the sweeping interval $\left[t_{0}, t_{1}\right]$. Let $\mathscr{R}_{\sigma}$ be the projection of $\sigma$ in the $t$-axis. Note that $\mathscr{R}_{\sigma} \subseteq\left[t_{0}, t_{1}\right]$.

For any slicing line, given by the parameter $t$, we define the following functions for use in the calibration function. Let $a(t)$ be the area enclosed by the figure and contained in the sweeping interval $\left[t_{0}, t\right]$ (that is, below the slicing line $t$ ). Thus $a\left(t_{0}\right)=0$ and $a\left(t_{1}\right)=A_{0}$ for any competitor. Let $l(t)$ be the total length of the intersection of the slicing line $t$ with the interior of $\sigma$ :

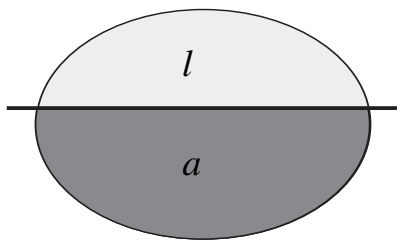

$\sigma$

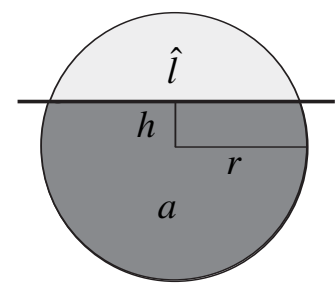

$\mu$

To complete our definition of the calibration function we define a map $F$ from a slicing line on the competitor to a slicing line on the conjectured minimizer, the circle enclosing area $A_{0}$. Let $r$ denote the radius of this circle. We also parametrize the circle with a set of parallel slicing lines. The position of these lines will be defined by the variable $h$, the $y$-coordinate of the lines, where $h=0$ passes through the center of the circle. Let $F(t)=h$ match the slicing line of the circle such that the area enclosed by the circle under the line $h$ is equal to $a(t)$. Let $\hat{l}$ be the length of the intersection of the slicing line $h$ with the interior of the circle. These functions are taken as $C^{1}$, since any competitor for which they are not may be shown to be nonminimal by a standard variational argument. While the functions defined above are all functions of the parameter $t$, we typically suppress the notation. 
Using the above functions we are now able to define the calibration function $G(\sigma)$.

Theorem 3.1. The function $G(\sigma)=\int_{\mathscr{R}_{\sigma}} f_{\sigma}^{\prime} d t$ calibrates the circle, where $f_{\sigma}(t)=$ $(2 a(t)-h(t) l(t)) / r$.

Proof of each condition for calibration will be given separately in Lemmas 3.2, 3.3, 3.4 .

Lemma 3.2. For $\mu$, the circle enclosing area $A_{0}, P(\mu)=G(\mu)$.

Proof. Note that $G(\mu)=\int_{\mathscr{R}_{\mu}} f^{\prime} d t=g\left(t_{1}\right)-g\left(t_{0}\right)=2 A_{0} / r+0$. Noting that in a circle $A_{0}=\pi r^{2}$, we find $2 A_{0} / r=2 \pi r^{2} / r=2 \pi r$, which is the perimeter of the circle. Thus $P(\mu)=G(\mu)$.

Lemma 3.3. For any competitor $\sigma \in S$ and the circle $\mu, G(\mu)=G(\sigma)$.

Proof. Note that since $\sigma$ may be disconnected with several components, $\mathscr{R}_{\sigma}=$ $\bigcup_{1}^{m}\left[x_{i}, y_{i}\right]$ where $x_{1}=t_{0}, y_{m}=t_{1}$, and $y_{i}<x_{i+1}$. Thus

$$
G(\sigma)=\int_{\Re_{\sigma}} f^{\prime} d t=\sum_{1}^{m} \int_{x_{i}}^{y_{i}} f^{\prime} d t=\sum_{1}^{m} f\left(y_{i}\right)-f\left(x_{i}\right) .
$$

Note that $l\left(x_{i}\right)=l\left(y_{i}\right)=0$ and $a\left(y_{i}\right)=a\left(x_{i+1}\right)$ for all $i$. Thus

$$
G(\sigma)=\sum_{1}^{m} f\left(y_{i}\right)-f\left(x_{i}\right)=\sum_{1}^{m} \frac{2 a\left(y_{i}\right)}{r}-\frac{2 a\left(x_{i}\right)}{r}=\frac{2 a\left(y_{m}\right)}{r}-\frac{2 a\left(x_{1}\right)}{r} .
$$

But $a\left(y_{m}\right)=a\left(t_{1}\right)=A_{0}$ and $a\left(x_{1}\right)=a\left(t_{0}\right)=0$. This implies that $G(\sigma)=2 A_{0} / r$, which is constant among all competitors in $S$, including $\mu$. Thus $G(\mu)=G(\sigma)$ for all $\sigma \in S$.

Lemma 3.4. For any competitor $\sigma, G(\sigma) \leq P(\sigma)$.

Proof. Differentiating $f$ by $t$ we find $f^{\prime}=\left(2 A^{\prime}-h^{\prime} l-h l^{\prime}\right) / r$. Now from the definition of $A$ and of $h$ we can see that $A^{\prime}=l$ in the competitor $\sigma$ and $A^{\prime}=h^{\prime} \hat{l}$ in the conjectured minimizer $\mu$. Substitution reveals

$$
f^{\prime}=\frac{\left(2-h^{\prime}\right) l-h l^{\prime}}{r}=\frac{\left(2-h^{\prime}\right) h^{\prime} \hat{l}-h l^{\prime}}{r} .
$$

Noting that $\left(2-h^{\prime}\right) h^{\prime}$ has a maximum at 1 for $h^{\prime}=1$, we find

$$
f^{\prime} \leq \frac{\hat{l}-h l^{\prime}}{r}=\frac{1}{r}\left(\frac{\hat{l}}{2},-h\right) \cdot\left(2, l^{\prime}\right) .
$$

Now $\frac{1}{r}\left(\frac{\hat{l}}{2},-h\right)$ is a unit vector in the circle $\mu$, so by the Cauchy-Schwartz inequality,

$$
f^{\prime} \leq\left\|\left(2, l^{\prime}\right)\right\|=2 \sqrt{1+\left(l^{\prime} / 2\right)^{2}} .
$$


Since the slicing line at height $t$ will intersect the figure at least twice if $l \neq 0$, $2 \sqrt{1+\left(l^{\prime} / 2\right)^{2}}$ realizes the minimum of $P^{\prime}$ via a symmetrization argument. Thus $f^{\prime} \leq P^{\prime}(\sigma)$, and since $f_{\sigma}(0)=P_{\sigma}(0)=0$, this implies $G(\sigma)=\int_{\mathscr{R}_{\sigma}} g^{\prime} d t \leq$ $\int_{\mathscr{R}_{\sigma}} P^{\prime}(\sigma) d t=P(\sigma)$.

Lemmas 3.2, 3.3, and 3.4 show that the function $G(\sigma)$ defined in Theorem 3.1 calibrates the circle. Thus by the calibration theorem, the circle minimizes perimeter of all $C^{1}$ manifolds enclosing a fixed area. Similar proofs that the sphere and $n$-sphere are boundary-minimizing in their respective dimensions have been found by the authors. In this paper, however, we investigate generalizations to multiple bubbles in two dimensions. The next section uses the methods of metacalibration to prove perimeter minimization of the standard double bubble in $\mathbb{R}^{2}$.

\section{The standard double bubble is perimeter-minimizing in $\mathbb{R}^{2}$}

The double bubble conjecture in $\mathbb{R}^{2}$ was first proved by students of Frank Morgan in an NSF funded REU in 1990 [Foisy et al. 1993]. They showed that the way to separately enclose two given areas with the least perimeter is the "standard double bubble," a figure with three circular arcs meeting two vertices at 120 angles. Here we present a new independent proof of the double bubble conjecture using the method of metacalibration.

Theorem 4.1. The standard double bubble in $\mathbb{R}^{2}$ minimizes total perimeter of figures (unions of $C^{1}$ manifolds) enclosing two separate fixed areas.

This will result from the calibration theorem using the calibration function defined below.

As with the calibration for the circle, we use a map from slices of the competitor to slices of our conjectured minimizer, the standard double bubble.

Each competitor double bubble $\sigma \in S$ will consist of two enclosed regions $B_{1}$ and $B_{2}$, with fixed areas $A_{1}$ and $A_{2}$. For a given competitor $\sigma$, parametrize parallel lines traversing the figure with the variable $t$. We let $a_{i}(t)$ be the area of $B_{i}$ below the line $t$, and let $l_{i}(t)$ be the length of the intersection of the line $t$ with $B_{i}$.

There is a unique standard double bubble that separately encloses areas $A_{1}$ and $A_{2}$ [Foisy et al. 1993]. Let $\hat{B}_{1}$ and $\hat{B}_{2}$ be the regions of this standard double bubble (of areas $A_{1}$ and $A_{2}$, respectively), and let $r_{1}$ and $r_{2}$ be the radii of the outer arcs bordering $\hat{B}_{1}$ and $\hat{B}_{2}$. Without loss of generality we assume that $r_{1} \geq r_{2}$, or equivalently $A_{1} \geq A_{2}$. We denote the radius of the arc separating $\hat{B}_{1}$ and $\hat{B}_{2}$ by $r_{3}$. We also denote the centers of each of these three arcs as $o_{1}, o_{2}$, and $o_{3}$ respectively. It is known that $1 / r_{3}=1 / r_{2}-1 / r_{1}$ for all standard double bubbles [Isenberg 1978 , pp. 88-95]. We also use the parameter $h$ to define slices of the standard double bubble. However, in order to map slicing lines $t$ in the competitor to slicing lines 
in the standard bubble, matching both areas as before, slicing lines in the standard bubble must be allowed to tilt. Thus we parametrize slicing lines in the standard double bubble with two variables: $h_{1}$ and $h_{2}$, the signed distance from the slicing line to $o_{1}$ and $o_{2}$, respectively $\left(h_{i}<0\right.$ if the line passes below $\left.o_{i}\right)$. As with the competitor, we let $\hat{a}_{i}(t)$ be the area of $\hat{B}_{i}$ below the line $\left(h_{1}, h_{2}\right)$, and let $\hat{l}_{i}(t)$ be the length of the intersection of the line $\left(h_{1}, h_{2}\right)$ with $\hat{B}_{i}$ :

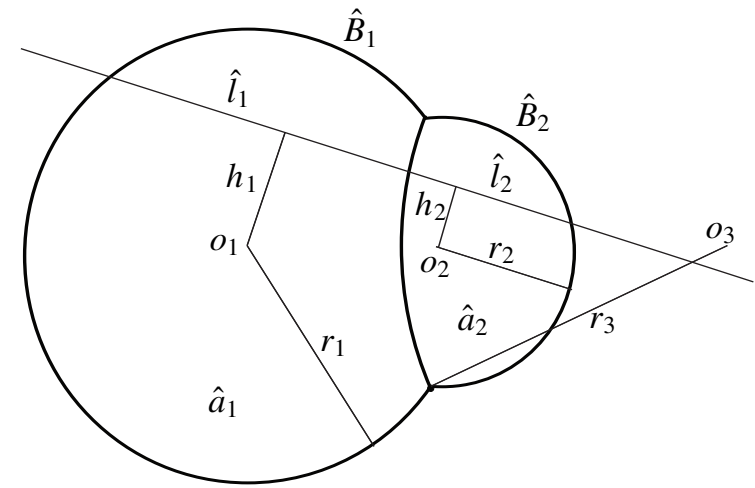

Each slice of a competitor defines a point $\left(a_{1}, a_{2}\right) \in\left[0, A_{1}\right] \times\left[0, A_{2}\right]$. In this sense, each competitor $\sigma$, when parametrized by $t$, describes a path $\sigma:[0,1] \rightarrow$ $\left[0, A_{1}\right] \times\left[0, A_{2}\right]$ such that $\sigma(0)=(0,0), \sigma(1)=\left(A_{1}, A_{2}\right)$ and $\sigma(t)=\left(a_{1}(t), a_{2}(t)\right)$.

We will define a map $F:\left[0, A_{1}\right] \times\left[0, A_{2}\right] \rightarrow\left[-r_{1}, r_{1}\right] \times\left[-r_{2}, r_{2}\right]$ between slices of the competitor and slices of the standard double bubble where $F\left(a_{1}, a_{2}\right)=$ $\left(h_{1}, h_{2}\right)$. We will also define $F$ such that for all $\left(a_{1}, a_{2}\right) \in\left[0, A_{1}\right] \times\left[0, A_{2}\right]$,

$$
a_{i}=\hat{a}_{i}\left(F\left(a_{1}, a_{2}\right)\right) .
$$

There are, however, some points in $\left[0, A_{1}\right] \times\left[0, A_{2}\right]$ that do not have such a map into the double bubble. For example, if $A_{1} \neq A_{2}$, no slice $\left(h_{1}, h_{2}\right)$ of the standard double bubble gives rise to $\left(\hat{a}_{1}, \hat{a}_{2}\right)=\left(A_{1}, 0\right)$. Thus we will need to restrict $F$ to some $K \subseteq\left[0, A_{1}\right] \times\left[0, A_{2}\right]$ such that $\left.F\right|_{K}$ exists and is one-to-one.

To define $K$, we first define $\mathbb{\complement} \subset\left[-r_{1}, r_{1}\right] \times\left[-r_{2}, r_{2}\right]$ which will be the image of $K$ under $F$. Let

$$
\mathbb{H}=\left\{\left(h_{1}, h_{2}\right) \in\left[-r_{1}, r_{1}\right] \times\left[-r_{2}, r_{2}\right] \text { such that }\left|h_{1}-h_{2}\right| \leq\left|o_{1}-o_{2}\right|\right\},
$$

where $\left|o_{1}-o_{2}\right|$ is the distance between the centers $o_{1}$ and $o_{2}$. This limits, by standard geometric properties, the parametrization $\left(h_{1}, h_{2}\right)$ to slices that are realizable on the standard double bubble. Let $E:\left[-r_{1}, r_{1}\right] \times\left[-r_{2}, r_{2}\right] \rightarrow\left[0, A_{1}\right] \times\left[0, A_{2}\right]$ be the function that takes a slicing line in the standard double bubble and returns

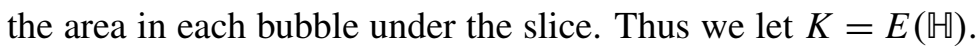


Lemma 4.2. The map $F=E^{-1}: K \rightarrow \mathbb{M}$ exists, is one-to-one, and is continuously differentiable.

Proof. Proof follows by application of the inverse function theorem on $F^{-1}$.

Consider $E: \operatorname{int}(\mathbb{M}) \rightarrow \operatorname{int}(K)$ where $E\left(h_{1}, h_{2}\right)=\left(\hat{a}_{1}, \hat{a}_{2}\right)$. Note that the map $E$ is continuously differentiable. Note also that

$$
D E=\left[\begin{array}{ll}
\frac{\partial a_{1}}{\partial h_{1}} & \frac{\partial a_{1}}{\partial h_{2}} \\
\frac{\partial a_{2}}{\partial h_{1}} & \frac{\partial a_{2}}{\partial h_{2}}
\end{array}\right] .
$$

To show that $D E$ is invertible, we merely need to show that $\operatorname{det}(D E) \neq 0$, or that

$$
\frac{\partial a_{1}}{\partial h_{1}} \frac{\partial a_{2}}{\partial h_{2}} \neq \frac{\partial a_{2}}{\partial h_{1}} \frac{\partial a_{1}}{\partial h_{2}} .
$$

It is easy to see from the figure below that, as $h_{i}$ increases, so must $a_{i}$; therefore $\partial a_{i} / \partial h_{i}>0$.

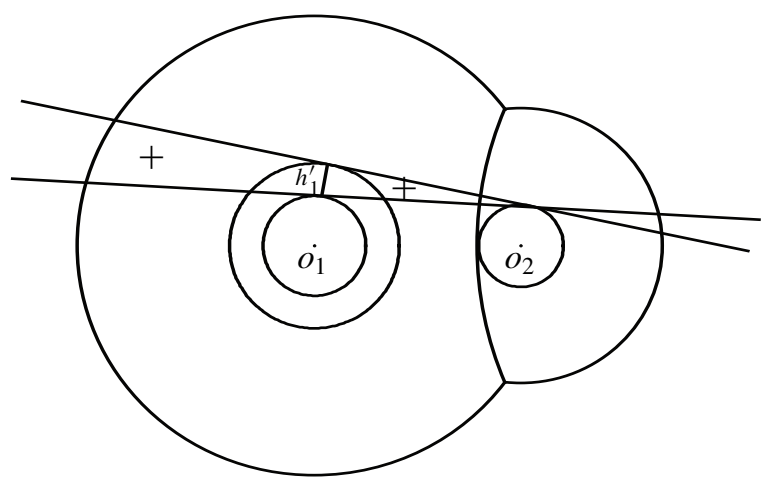

Claim. When $h_{2}$ increases, $\partial a_{1} / \partial h_{2} \leq 0$ and $\left|\partial a_{1} / \partial h_{2}\right|<\left|\partial a_{1} / \partial h_{1}\right|$.

Proof. Let $B_{1}$ be a single bubble, as in the figure:

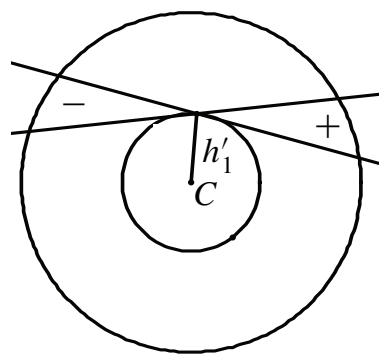

Note that $h_{1}$ is constant here. Since the slicing line must be tangent to the circle centered at $o_{1}$ with a radius of $h_{1}$, both slicing lines must intersect at a point, losing area on the left and gaining area on the right. By basic trigonometry, these 
two areas will be additive inverses. Now if we add $B_{2}$, some of the area on the right is lost to $B_{2}$, like this:

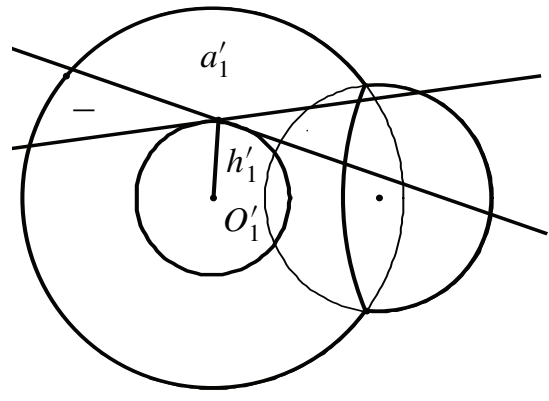

So as $h_{2}$ increases, $a_{1}$ will lose more area than it gains, making $\partial a_{1} / \partial h_{2} \leq 0$. Note also that $\left|\partial a_{1} / \partial h_{2}\right|$, the area lost by adding $B_{2}$, represents a subset of the area gained by $a_{2}$ as $h_{2}$ increases $\left(\left|\partial a_{2} / \partial h_{2}\right|\right)$. So $\left|\partial a_{1} / \partial h_{2}\right|<\left|\partial a_{1} / \partial h_{1}\right|$.

We claim by the same method that $\partial a_{2} / \partial h_{1} \leq 0$ and $\left|\partial a_{2} / \partial h_{1}\right|<\left|\partial a_{2} / \partial h_{2}\right|$.

It follows that $\left(\partial a_{1} / \partial h_{1}\right)\left(\partial a_{2} / \partial h_{2}\right)$ and $\left(\partial a_{1} / \partial h_{2}\right)\left(\partial a_{2} / \partial h_{1}\right)$ are always nonnegative,

$$
\left(\partial a_{1} / \partial h_{2}\right)\left(\partial a_{2} / \partial h_{2}\right)<\left(\partial a_{1} / \partial h_{1}\right)\left(\partial a_{2} / \partial h_{2}\right),
$$

and $\operatorname{det}(D E) \neq 0$. Hence $D E$ is invertible everywhere on int $(\llbracket)$.

Thus the inverse function theorem implies that $E$ is locally a bijection $\left(F=E^{-1}\right.$ exists) and that $F$ is continuously differentiable. The map $F$ is also one-to-one since $F\left(a_{1}, a_{2}\right)=F\left(b_{1}, b_{2}\right)=\left(h_{1}, h_{2}\right)$ implies $\left(a_{1}, a_{2}\right)=\left(b_{1}, b_{2}\right)=E\left(h_{1}, h_{2}\right)$.

We complete the proof of Lemma 4.2 by showing that $F=E^{-1}: \partial K \rightarrow \partial \mathbb{U}$ exists and is one-to-one. We do this by describing a smooth bijection from $\partial K$ onto $\partial$ 円H.

For the slicing line to be on the boundary of $K$, it must be tangent to the boundary of at least one of the bubbles (save on the extremes where $\left|h_{1}-h_{2}\right|=\left|o_{1}-o_{2}\right|$ ). Imagine the line tangent to both bubbles on the bottom, marked $a$ in the figure:
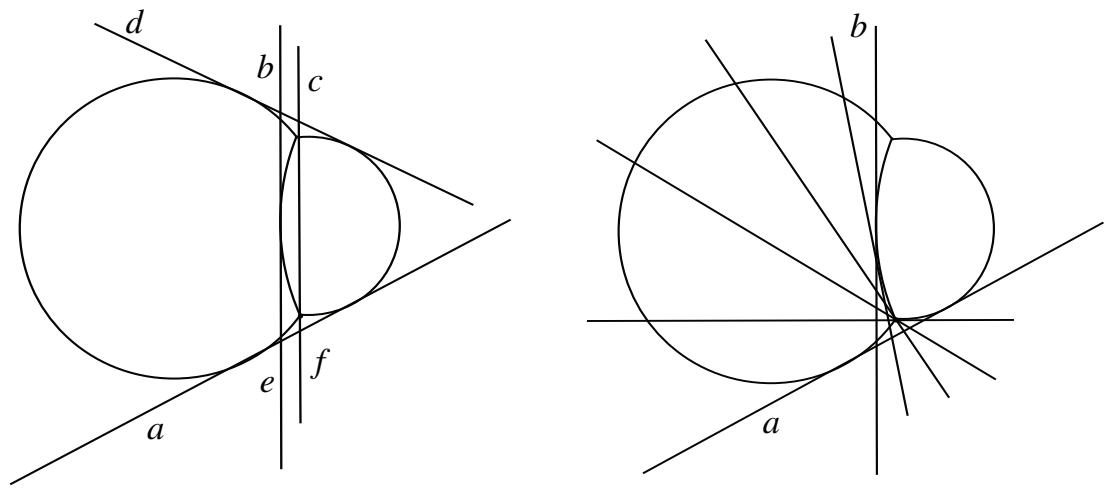
We'll move the line upward in bubble 1, keeping the line tangent to the bottom of bubble 2, until we reach line $b$, where it is vertical, touching the left side of bubble 2. It then slides horizontally to the right to touch the vertices of the bubbles (line $c$ ). The line will rotate upwards in bubble 2 while touching the boundary of bubble 1 until it is tangent to the top of both bubbles (line $d$ ). Then the line will stay touching the boundary of bubble 2 and rotate down through bubble 1 until it is once again vertical touching the boundary (line $e$ ). It will shift right until it touches the intersections (line $f$ ). Finally, the line will touch bubble 1's boundary and move down through bubble 2 until reaching the bottom of both bubbles and the starting point (line $a$ ). In this way, we've smoothly and bijectively traversed all of the slicing lines that are on the boundary of $K$. (Note that slicing lines are of necessity oriented, as each slicing line has a defined region below the line.)

This implies that if $\sigma(t) \in K$ for all $t \in[0,1], F(\sigma(t))$ describes a continuously differentiable path in $\mathbb{W}$ where $\left.\left(\hat{a}_{1}, \hat{a}_{2}\right)\right|_{F(\sigma(t))}=\sigma(t)$. To ensure that $\sigma(t)$ is always in the domain of $F$, we place the following restriction on the parametrizations of a competitor $\sigma$. Let $t_{0}$ denote the line that passes through the centroids of $B_{1}$ and $B_{2}$. (If these coincide, any line passing through them is sufficient.) Parametrize the competitor $\sigma$ such that all slicing lines are parallel to $t_{0}$. Since $t_{0}$ passes through the centroids of $B_{1}$ and $B_{2}$, it cuts their areas exactly in half, and $\sigma\left(t_{0}\right)=\left(A_{1} / 2, A_{2} / 2\right)$ for all competitors $\sigma$. Now $\sigma(t)$ is nondecreasing in both $a_{1}$ and $a_{2}$, so $\sigma(t) \in$ $\left[0, A_{1} / 2\right] \times\left[0, A_{2} / 2\right] \cup\left[A_{1} / 2, A_{1}\right] \times\left[A_{2} / 2, A_{2}\right] \subset K$ for all $t$. confining it to the white part of the figure. Hence the mapping $F$ may be applied to any competitor $\sigma$ given this orientation of slicing lines.

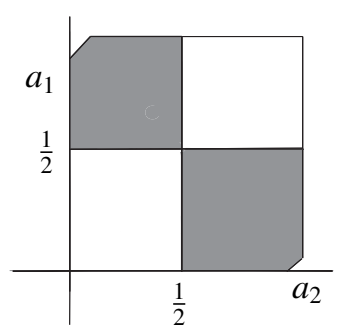

This map allows the following definition of $G(\sigma)$.

Theorem 4.3. Let $f_{\sigma}=\sum\left(2 A_{i}-h_{i} l_{i}\right) / r_{i}$ for $i=1,2$. The function $G(\sigma)=$ $\int_{t_{0}}^{t_{1}} f_{\sigma}^{\prime} d t$ calibrates the standard double bubble.

We prove the three conditions for a calibration function separately in Lemmas 4.4, 4.5, and 4.10.

Lemma 4.4. $G(\mu)=P(\mu)$ for $\mu$ a standard double bubble. 
Proof. Consider the vector fields $\boldsymbol{V}=(x, y) / r_{1}$ and $\boldsymbol{W}=(x-m, y) / r_{2}$, where $(0,0)=o_{1}$ and $(m, 0)=o_{2}$. We claim the total perimeter of a standard double bubble, $P(\mu)$, is equal to

$$
\int_{\partial \hat{B}_{1}} \boldsymbol{V} \cdot \boldsymbol{N} d s+\int_{\partial \hat{B}_{2}} \boldsymbol{W} \cdot \boldsymbol{N} d s
$$

where $N$ is the unit normal to the surface at that point. To see this, note that on the outer arcs of radius $r_{1}$ and $r_{2}, \boldsymbol{V}=\boldsymbol{N}$ and $\boldsymbol{W}=\boldsymbol{N}$, and the integral over these arcs reduces to $\int 1 d s$. The integral over the center $\operatorname{arc}$ reduces to $\int(\boldsymbol{W}-\boldsymbol{V}) \cdot \boldsymbol{N} d s$, with the appropriate normal vector. However,

$$
\begin{aligned}
\boldsymbol{W}-\boldsymbol{V} & =\frac{1}{r_{2}}(x-m, y)-\frac{1}{r_{1}}(x, y)=\left(\frac{1}{r_{2}}-\frac{1}{r_{1}}\right)(x, y)-\frac{1}{r_{2}}(m, 0) \\
& =\frac{1}{r_{3}}(x, y)-\frac{1}{r_{2}}(m, 0)=\frac{1}{r_{3}}\left(x-\frac{r_{3}}{r_{2}} m, y\right) .
\end{aligned}
$$

If we denote the intersection of the three arcs in a standard double bubble by $p$, we see by the fact that these arcs meet at $120^{\circ}$ angles that $m \angle o_{1} p o_{2}=60^{\circ}$ and $m \angle o_{1} p o_{3}=120^{\circ}$. By application of the law of sines we find that

$$
\frac{\left|o_{1}-o_{2}\right|}{\sin 60^{\circ}}=\frac{r_{2}}{\sin \left(m \angle o_{2} o_{1} p\right)} \quad \text { and } \quad \frac{\left|o_{1}-o_{3}\right|}{\sin 120^{\circ}}=\frac{r_{3}}{\sin \left(m \angle o_{2} o_{1} p\right)}
$$

which reduces to

$$
\frac{\left|o_{1}-o_{2}\right|}{r_{2} \sin 60^{\circ}}=\frac{\left|o_{1}-o_{3}\right|}{r_{3} \sin 120^{\circ}} \frac{r_{3}\left|o_{1}-o_{2}\right|}{r_{2}}=\left|o_{1}-o_{3}\right| \frac{r_{3}}{r_{2}} m=\left|o_{1}-o_{3}\right| .
$$

Thus $o_{3}=\left(\frac{r_{3}}{r_{2}} m, 0\right)$, and the vector $\boldsymbol{W}-\boldsymbol{V}=\frac{1}{r_{3}}\left(x-\frac{r_{3}}{r_{2}} m, y\right)$ is equal to the unit normal on the remaining arc. Thus the line integral on this arc also evaluates to $\int 1 d s$. Since the line integrals over all three arcs evaluate to $\int 1 d s$, or the length of each arc, the expression in (1) above is equal to $P(\mu)$. As a consequence of the divergence theorem, however, we have

$$
\begin{aligned}
P(\mu) & =\int_{\partial \hat{B}_{1}} \boldsymbol{V} \cdot \boldsymbol{N} d s+\int_{\partial \hat{B}_{2}} \boldsymbol{W} \cdot \boldsymbol{N} d s=\int_{\hat{B}_{1}} \operatorname{div} \boldsymbol{V} d A+\int_{\hat{B}_{2}} \operatorname{div} \boldsymbol{W} d A \\
& =\int_{\hat{B}_{1}} \frac{\partial}{\partial x}\left(\frac{x}{r_{1}}\right)+\frac{\partial}{\partial y}\left(\frac{y}{r_{1}}\right) d A+\int_{\hat{B}_{2}} \frac{\partial}{\partial x}\left(\frac{x-m}{r_{2}}\right)+\frac{\partial}{\partial y}\left(\frac{y}{r_{2}}\right) d A \\
& =\int_{\hat{B}_{1}} \frac{1}{r_{1}}+\frac{1}{r_{1}} d A+\int_{\hat{B}_{2}} \frac{1}{r_{2}}+\frac{1}{r_{2}} d A=\int_{\hat{B}_{1}} \frac{2}{r_{1}} d A+\int_{\hat{B}_{2}} \frac{2}{r_{2}} d A=\frac{2 A_{1}}{r_{1}}+\frac{2 A_{2}}{r_{2}} .
\end{aligned}
$$


Now consider $G(\sigma)$ :

$$
\begin{aligned}
G(\sigma) & =\int_{0}^{1} f_{\sigma}^{\prime} d t=f_{\sigma}(1)-f_{\sigma}(0)=\left.\sum \frac{2 a_{i}-h_{i} l_{i}}{r_{i}}\right|_{t=1}-\left.\sum \frac{2 a_{i}-h_{i} l_{i}}{r_{i}}\right|_{t=0} \\
& =\sum \frac{2 A_{i}-1 \cdot 0}{r_{i}}-\sum \frac{2 \cdot 0-1 \cdot 0}{r_{i}}=\frac{2 A_{1}}{r_{1}}+\frac{2 A_{2}}{r_{2}} .
\end{aligned}
$$

This is true for all competitors $\sigma$, including the standard double bubble $\mu$; thus $P(\mu)=G(\mu)$.

Lemma 4.5. $G(\sigma)=G(\mu)$ for all competitors $\sigma$, where $\mu$ is the standard double bubble.

Proof. It was shown in Lemma 4.4 that $G(\sigma)=2 A_{1} / r_{1}+2 A_{2} / r_{2}$ for all competitors $\sigma$, including $\mu$. Thus $G(\sigma)=G(\mu)$ for all competitors $\sigma$.

The final condition for calibration, that $G(\sigma) \leq P(\sigma)$ for all competitors $\sigma$, will be proved in Lemma 4.10 as a result of the following propositions. For this section we introduce the notation $G_{\sigma}(t)=\int_{t_{0}}^{t} f_{\sigma}^{\prime} d t$ and $P_{\sigma}(t)=$ the total perimeter of $\sigma$ lying below the slicing line $t$. We will show that $G_{\sigma}^{\prime}(t) \leq P_{\sigma}^{\prime}(t)$ for all $t \in\left[t_{0}, t_{1}\right]$, and $G(\sigma) \leq P(\sigma)$ will result by integration.

In the following propositions we will use the notation shown in these figures:

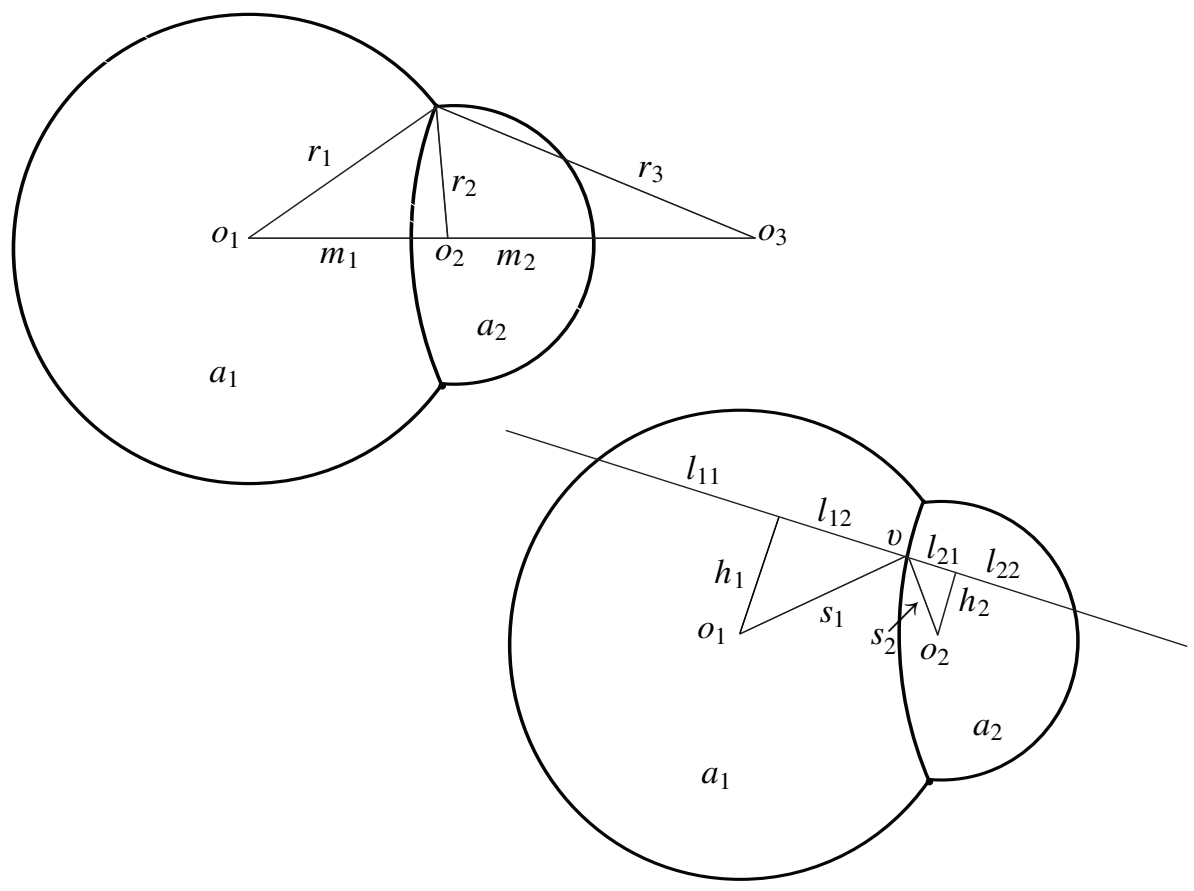

Let $m_{1}=\left|o_{1}-o_{2}\right|$ (the $m$ of Lemma 4.4) and $m_{2}=\left|o_{2}-o_{3}\right|$. Also let $v$ be the intersection of the slicing line $\left(h_{1}, h_{2}\right)$ with the center arc between $\hat{B}_{1}$ and $\hat{B}_{2}$. 
Define $s_{1}, s_{2}$, as the distances between $v$ and $o_{1}, o_{2}$ respectively. The lengths $l_{11}$, $l_{12}, l_{21}$, and $l_{22}$ are defined as shown, where $l_{11}+l_{12}=\hat{l}_{1}$ and $l_{21}+l_{22}=\hat{l}_{2}$.

Proposition 4.6. For all slices $\left(h_{1}, h_{2}\right)$ of the standard double bubble,

$$
\frac{l_{11}^{2}-l_{12}^{2}}{r_{1}}=\frac{l_{21}^{2}-l_{22}^{2}}{r_{2}} \text {. }
$$

Proof. It was shown in Lemma 4.4 that $o_{1} o_{3}=m_{1}+m_{2}=\left(r_{3} / r_{2}\right) m_{1}$. Given that $r_{3}=r_{1} r_{2} /\left(r_{1}-r_{2}\right)$, we find that $m_{1} / m_{2}=\left(r_{1}-r_{2}\right) / r_{2}$. Noting that $\cos \left(\angle o_{1} o_{2} v\right)=$ $-\cos \left(\angle v o_{2} o_{3}\right)$, by application of the law of cosines we find

$$
\frac{m_{1}^{2}+s_{2}^{2}-s_{1}^{2}}{2 s_{2} m_{1}}=-\frac{s_{2}^{2}+m_{2}^{2}-r_{3}^{2}}{2 s_{2} m_{2}},
$$

from which we obtain, successively,

$$
\begin{aligned}
-s_{2}^{2}\left(1+\frac{m_{1}}{m_{2}}\right) & =m_{1}^{2}+\frac{m_{1}}{m_{2}}\left(m_{2}^{2}-r_{3}^{2}\right)-s_{1}^{2}, \\
-s_{2}^{2}\left(1+\frac{r_{1}-r_{2}}{r_{2}}\right) & =r_{1}^{2}+r_{2}^{2}-r_{1} r_{2}+\frac{r_{1}-r_{2}}{r_{2}}\left(r_{2}^{2}+r_{3}^{2}-r_{2} r_{3}-r_{3}^{2}\right)-s_{1}^{2}, \\
-s_{2}^{2} \frac{r_{1}}{r_{2}} & =r_{1}^{2}+r_{2}^{2}-r_{1} r_{2}+\frac{r_{1}-r_{2}}{r_{2}}\left(r_{2}^{2}-r_{2} \frac{r_{1} r_{2}}{r_{1}-r_{2}}\right)-s_{1}^{2}, \\
r_{1} r_{2}-s_{2}^{2} \frac{r_{1}}{r_{2}} & =r_{1}^{2}+r_{2}^{2}+\frac{r_{1}-r_{2}}{r_{2}} \frac{-r_{2}^{3}}{r_{1}-r_{2}}-s_{1}^{2}=r_{1}^{2}-s_{1}^{2}, \\
\frac{r_{2}^{2}-s_{2}^{2}}{r_{2}} & =\frac{r_{1}^{2}-s_{1}^{2}}{r_{1}} .
\end{aligned}
$$

Now by the Pythagorean theorem we find $l_{21}^{2}-l_{22}^{2}=r_{2}^{2}-h_{2}^{2}-\left(s_{2}^{2}-h_{2}^{2}\right)=r_{2}^{2}-s_{2}^{2}$ and $l_{11}^{2}-l_{12}^{2}=r_{1}^{2}-h_{1}^{2}-\left(s_{1}^{2}-h_{1}^{2}\right)=r_{1}^{2}-s_{1}^{2}$. Substitution shows that $\left(l_{11}^{2}-l_{12}^{2}\right) / r_{1}=$ $\left(l_{21}^{2}-l_{22}^{2}\right) / r_{2}$.

Proposition 4.7. $\sum\left(\left(2-h_{i}^{\prime}\right) a_{i}^{\prime}\right) / r_{i} \leq \sum \hat{l}_{i} / r_{i}$.

Proof. Let $l_{11}, l_{12}, l_{21}, l_{22}, h_{1}$, and $h_{2}$ be defined as above. Consider the diagram

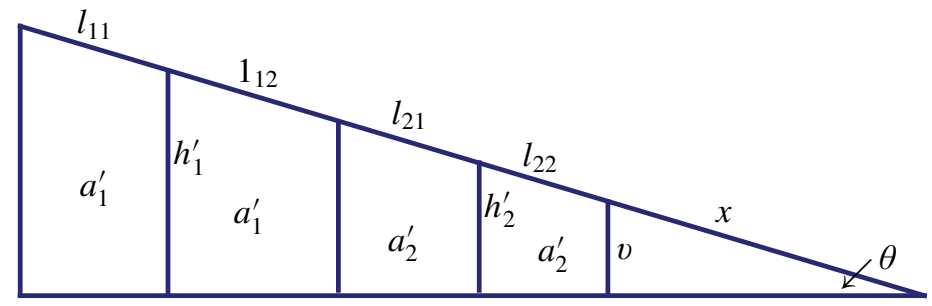

which describes the instantaneous change in $\left(h_{1}, h_{2}\right)$. Unlike the parametrizations of slicing lines in the circle, in the standard double bubble slicing lines may rotate. 
This rotation is described by the relative angle $\theta^{\prime}$ and the distance $x$ between the axis of rotation and the double bubble.

We can compute the total change in area in both $\hat{B}_{1}$ and $\hat{B}_{2}$ as follows:

$$
\begin{aligned}
& A_{1}^{\prime}=\frac{1}{2} \theta^{\prime}\left(\left(x+\hat{l}_{2}+\hat{l}_{1}\right)^{2}-\left(x+\hat{l}_{2}\right)^{2}\right)=\frac{1}{2} \theta^{\prime}\left(2 x+2 \hat{l}_{2}+\hat{l}_{1}\right)\left(\hat{l}_{1}\right), \\
& A_{2}^{\prime}=\frac{1}{2} \theta^{\prime}\left(\left(x+\hat{l}_{2}\right)^{2}-(x)^{2}\right)=\frac{1}{2} \theta^{\prime}\left(2 x+\hat{l}_{2}\right)\left(\hat{l}_{2}\right) .
\end{aligned}
$$

We also note that $h_{1}^{\prime}=\theta^{\prime}\left(x+\hat{l}_{2}+l_{12}\right)$ and $h_{2}^{\prime}=\theta^{\prime}\left(x+l_{22}\right)$. Thus

$$
\begin{aligned}
\sum \frac{\left(2-h_{i}^{\prime}\right) A_{i}^{\prime}}{r_{i}} & \\
= & \frac{\left(2-h_{1}^{\prime}\right)\left(\theta^{\prime} / 2\right)\left(2 x+2 \hat{l}_{2}+\hat{l}_{1}\right)\left(\hat{l}_{1}\right)}{r_{1}}+\frac{\left(2-h_{2}^{\prime}\right)\left(\theta^{\prime} / 2\right)\left(2 x+\hat{l}_{2}\right)\left(\hat{l}_{2}\right)}{r_{2}} \\
= & \frac{\left(2-h_{1}^{\prime}\right) \frac{h_{1}^{\prime}}{2\left(x+\hat{l}_{2}+l_{12}\right)}\left(2 x+2 \hat{l}_{2}+\hat{l}_{1}\right)\left(\hat{l}_{1}\right)}{r_{1}}+\frac{\left(2-h_{2}^{\prime}\right) \frac{h_{2}^{\prime}}{2\left(x+l_{22}\right)}\left(2 x+\hat{l}_{2}\right)\left(\hat{l}_{2}\right)}{r_{2}} .
\end{aligned}
$$

Both of these components are maximized when $h_{1}^{\prime}=h_{2}^{\prime}=1$, so we have:

$$
\begin{aligned}
\sum \frac{\left(2-h_{i}^{\prime}\right) A_{i}^{\prime}}{r_{i}} & \leq \frac{\left(2 x+2 \hat{l}_{2}+\hat{l}_{1}\right)\left(\hat{l}_{1}\right)}{2\left(x+\hat{l}_{2}+l_{12}\right) r_{1}}+\frac{\left(2 x+\hat{l}_{2}\right)\left(\hat{l}_{2}\right)}{2\left(x+l_{22}\right) r_{2}} \\
& =\frac{\hat{l}_{1}}{r_{1}}+\frac{\hat{l}_{2}}{r_{2}}+\frac{\left(l_{11}-l_{12}\right)\left(l_{12}+l_{11}\right)}{2\left(x+\hat{l}_{2}+l_{12}\right) r_{1}}+\frac{\left(l_{21}-l_{22}\right)\left(l_{22}+l_{21}\right)}{2\left(x+l_{22}\right) r_{2}} \\
& =\frac{\hat{l}_{1}}{r_{1}}+\frac{\hat{l}_{2}}{r_{2}}+\frac{l_{11}^{2}-l_{12}^{2}}{2\left(x+\hat{l}_{2}+l_{12}\right) r_{1}}+\frac{l_{21}^{2}-l_{22}^{2}}{2\left(x+l_{22}\right) r_{2}} .
\end{aligned}
$$

By Proposition 4.6 we find $\frac{l_{11}^{2}-l_{12}^{2}}{r_{1}}=\frac{l_{21}^{2}-l_{22}^{2}}{r_{2}}$. Using this substitution we find

$$
\begin{aligned}
\frac{l_{11}^{2}-l_{12}^{2}}{2\left(x+\hat{l}_{2}+l_{12}\right) r_{1}}+\frac{l_{21}^{2}-l_{22}^{2}}{2\left(x+l_{22}\right) r_{2}} & =\frac{l_{21}^{2}-l_{22}^{2}}{2 r_{2}}\left(\frac{-1}{x+\hat{l}_{2}+l_{12}}+\frac{1}{x+l_{22}}\right) \\
& =\frac{l_{21}^{2}-l_{22}^{2}}{2 r_{2}} \frac{l_{12}+l_{21}}{\left(x+\hat{l}_{2}+l_{12}\right)\left(x+l_{22}\right)} .
\end{aligned}
$$

Now since $x+l_{22}>0$ and $l_{22}>l_{21}$, this term is always negative, and maximized at zero as $a \rightarrow \infty$. Thus

$$
\begin{aligned}
\sum \frac{\left(2-h_{i}^{\prime}\right) A_{i}^{\prime}}{r_{i}} & \leq \frac{\hat{l}_{1}}{r_{1}}+\frac{\hat{l}_{2}}{r_{2}}+\frac{l_{21}^{2}-l_{22}^{2}}{2 r_{2}} \frac{l_{12}+l_{21}}{\left(x+\hat{l}_{2}+l_{12}\right)\left(x+l_{22}\right)} \\
& \leq \frac{\hat{l}_{1}}{r_{1}}+\frac{\hat{l}_{2}}{r_{2}}=\sum \frac{\hat{l}_{i}}{r_{i}} .
\end{aligned}
$$


Proposition 4.8. For all slices of a standard double bubble, we have

$$
\left(\frac{l_{12}}{r_{1}}+\frac{l_{21}}{r_{2}}\right)^{2}+\left(\frac{h_{1}}{r_{1}}-\frac{h_{2}}{r_{2}}\right)^{2}=1 .
$$

Proof. We will show that

$$
\frac{h_{1}}{r_{1}}-\frac{h_{2}}{r_{2}}=-\cos \eta \quad \text { and } \quad \frac{l_{12}}{r_{1}}+\frac{l_{21}}{r_{2}}=-\sin \eta,
$$

where the angle $\eta$ is defined in the figure:

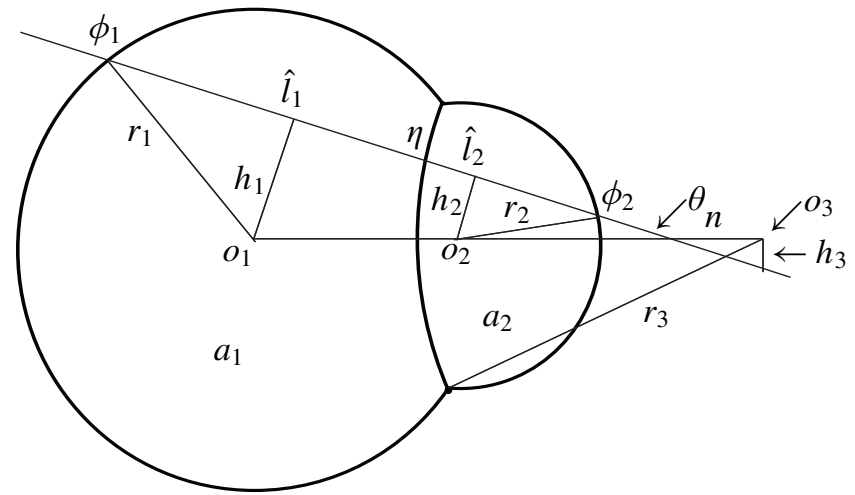

The proposition results from the equalities in (2). To show them, we prove that

$$
\cos \phi_{1}-\cos \phi_{2}+\cos \eta=0 .
$$

for any slice of a standard double bubble. Note that $\cos \phi_{1}=h_{1} / r_{1}, \cos \phi_{2}=h_{2} / r_{2}$, and $\cos \eta=h_{3} / r_{3}$. (Here $h_{3}$ is the signed distance from the slicing line to $o_{3}$, measured similarly to $h_{1}$ and $h_{2}$.) Let $n$ be the signed distance from $o_{3}$ to the intersection of the slicing line and the line through the centers, and $\theta$ the angle formed at the intersection, as in the figure above.

We find that $h_{1}=\left(m_{1}+m_{2}+n\right) \sin \theta, h_{2}=\left(m_{2}+n\right) \sin \theta$, and $h_{3}=n \sin \theta$. Hence

$$
\begin{aligned}
\cos \phi_{1}- & \cos \phi_{2}+\cos \eta \\
& =\frac{h_{1}}{r_{1}}-\frac{h_{2}}{r_{2}}+\frac{h_{3}}{r_{3}}=\frac{\left(m_{1}+m_{2}+n\right) \sin \theta}{r_{1}}-\frac{\left(m_{2}+n\right) \sin \theta}{r_{2}}+\frac{n \sin \theta}{r_{3}} \\
& =\left(r_{2}\left(m_{1}+m_{2}+n\right)-r_{1}\left(m_{2}+n\right)+\left(r_{1}-r_{2}\right) n\right) \frac{\sin \theta}{r_{1} r_{2}} \\
& =\left(r_{2}\left(\frac{m_{1}}{m_{2}}+1\right)-r_{1}\right) \frac{m_{2} \sin \theta}{r_{1} r_{2}}=\left(r_{2}\left(\frac{r_{1}-r_{2}}{r_{2}}+1\right)-r_{1}\right) \frac{m_{2} \sin \theta}{r_{1} r_{2}}=0 .
\end{aligned}
$$

This implies immediately that $h_{1} / r_{1}-h_{2} / r_{2}=-\cos \eta$. Now consider a slicing line perpendicular to the original at $v$, as in the figure on the top of the next page. In this slicing, we see that $l_{12} / r_{1}=\cos \phi_{1}$ and $l_{21} / r_{2}=-\cos \phi_{2}$. By the property just 


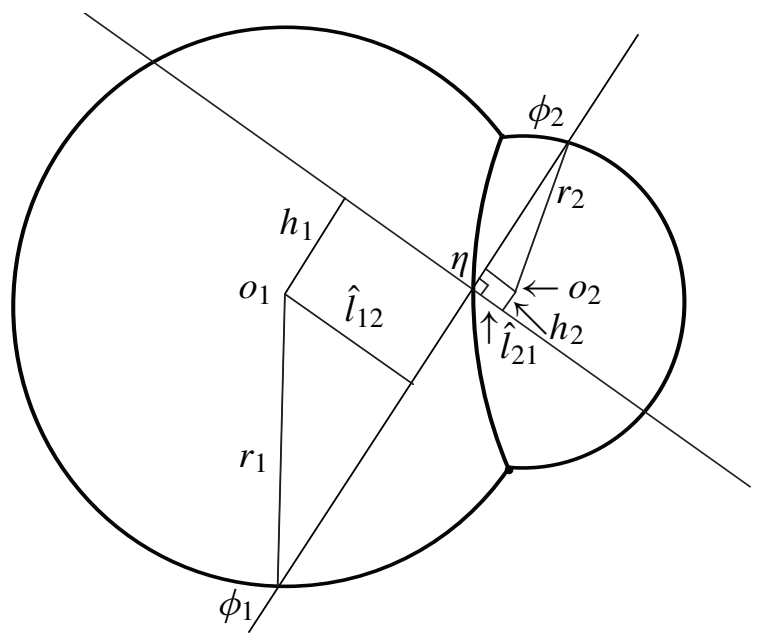

Towards the proof of Proposition 4.8.

proven, $\cos \phi_{1}-\cos \phi_{2}=-\cos \left(90^{\circ}+\eta\right)$, or equivalently $l_{12} / r_{1}+l_{21} / r_{2}=-\sin \eta$. These two relations together finish the proof.

Proposition 4.9. The following identity holds for all slices $t$ of any competitor $\sigma$ :

$$
\sqrt{1-\left(\frac{h_{1}}{r_{1}}\right)^{2}}+\sqrt{1-\left(\frac{h_{1}}{r_{1}}-\frac{h_{2}}{r_{2}}\right)^{2}}+\sqrt{1-\left(\frac{h_{2}}{r_{2}}\right)^{2}}+\sum \frac{-h_{i} l_{i}^{\prime}}{r_{i}} \leq P_{\sigma}^{\prime}(t) .
$$

Proof. Suppose the slicing line $t$ intersects $\sigma$ in three locations. Consider $P_{\sigma}^{\prime}(t)$, shown in the figure:

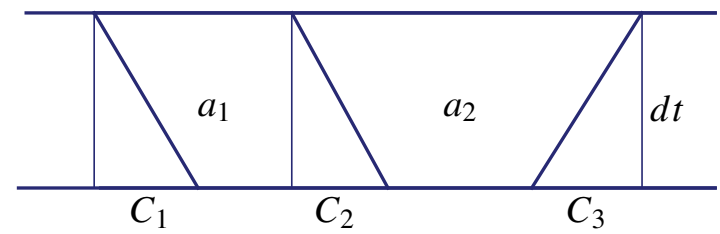

Note that $\left\|\left(c_{1}, d t\right)\right\|+\left\|\left(c_{2}, d t\right)\right\|+\left\|\left(c_{3}, d t\right)\right\|=P_{\sigma}^{\prime}(t) d t$. Now consider the unit vectors

$$
\left(-h_{1}, \sqrt{1-h_{1}^{2}}\right),\left(-h_{1}+h_{2}, \sqrt{1-\left(h_{1}-h_{2}\right)^{2}}\right),\left(-h_{2}, \sqrt{1-h_{2}^{2}}\right) .
$$

By the Cauchy-Schwartz inequality,

$$
\begin{array}{r}
\left(-h_{1}, \sqrt{1-h_{1}^{2}}\right) \cdot\left(c_{1}, d t\right)+\left(h_{2}-h_{1}, \sqrt{1-\left(h_{1}-h_{2}\right)^{2}}\right) \cdot\left(c_{2}, d t\right)+\left(-h_{2}, \sqrt{1-h_{2}^{2}}\right) \cdot\left(c_{3}, d t\right) \\
\leq\left\|\left(c_{1}, d t\right)\right\|+\left\|\left(c_{2}, d t\right)\right\|+\left\|\left(c_{3}, d t\right)\right\|=P_{\sigma}^{\prime}(t) d t .
\end{array}
$$

Noting that $\left(c_{1}+c_{2}\right) / d t=l_{1}^{\prime}$ and $\left(c_{3}-c_{2}\right) / d t=l_{2}^{\prime}$, this reduces to

$$
\sqrt{1-\left(\frac{h_{1}}{r_{1}}\right)^{2}}+\sqrt{1-\left(\frac{h_{1}}{r_{1}}-\frac{h_{2}}{r_{2}}\right)^{2}}+\sqrt{1-\left(\frac{h_{2}}{r_{2}}\right)^{2}}+\sum \frac{-h_{i} l_{i}^{\prime}}{r_{i}} \leq P_{\sigma}^{\prime}(t) .
$$


Now this inequality still holds even if $t$ intersects $\sigma$ in more than three locations by ignoring any additional intersections. There are however additional cases that are not covered by the above proof (such as when there are only two intersections), but for the sake of brevity we simply assert that proof of all other cases continues in much the same fashion.

Lemma 4.10. $G(\sigma) \leq P(\sigma)$ for all competitors $\sigma$.

Proof. We use the preceding propositions to show that $G_{\sigma}^{\prime}(t) \leq P_{\sigma}^{\prime}(t)$ for all $t \in[0,1]:$

$$
\begin{aligned}
G_{\sigma}^{\prime}(t) & =\sum \frac{2 A_{i}^{\prime}-h_{i}^{\prime} l_{i}-h_{i} l_{i}^{\prime}}{r_{i}}=\sum \frac{\left(2-h_{i}^{\prime}\right) A_{i}^{\prime}-h_{i} l_{i}^{\prime}}{r_{i}} \\
& \leq \sum \frac{\hat{l}_{i}-h_{i} l_{i}^{\prime}}{r_{i}} \quad \text { (by Proposition 4.7) } \\
& =\frac{l_{11}}{r_{1}}+\frac{l_{12}}{r_{1}}+\frac{l_{21}}{r_{2}}+\frac{l_{22}}{r_{2}}+\sum \frac{-h_{i} l_{i}^{\prime}}{r_{i}} \\
& =\sqrt{1-\left(\frac{h_{1}}{r_{1}}\right)^{2}}+\sqrt{1-\left(\frac{h_{1}}{r_{1}}-\frac{h_{2}}{r_{2}}\right)^{2}}+\sqrt{1-\left(\frac{h_{2}}{r_{2}}\right)^{2}}+\sum \frac{-h_{i} l_{i}^{\prime}}{r_{i}} \\
\quad & \quad \text { (by the Pythagorean theorem and Proposition 4.8) } \\
& \leq P_{\sigma}^{\prime}(t) \quad \text { (by Proposition 4.9). }
\end{aligned}
$$

Noting that $P_{\sigma}(0)=0$, we complete the proof by integration: $G_{\sigma}^{\prime}(t) \leq P_{\sigma}^{\prime}(t)$, so

$$
\int_{0}^{1} G_{\sigma}^{\prime}(t) d t \leq \int_{0}^{1} P_{\sigma}^{\prime}(t) d t
$$

and therefore $G(\sigma) \leq P_{\sigma}(1)-P_{\sigma}(0)$ and $G(\sigma) \leq P(\sigma)$.

This completes the proof of Theorem 4.3, namely that the standard double bubble minimizes perimeter among all figures (unions of $C^{1}$ manifolds) that separately enclose two fixed areas.

\section{Further research}

Extending the work that we have here presented seems well within the grasp of many undergraduate researchers. We are currently working on extending it to include many other such problems including soap films on wire frames, both with and without trapped bubbles. These problems are uniquely suited to metacalibrations because they include both fixed volume and fixed boundary constraints. The planar problem for three or more bubbles has proven somewhat more difficult to tackle, in large part because we no longer have the topological property of being able to match areas under a given slicing line, which was possible with two areas. Alternative slicing methods are being investigated to attack this problem. 
Another interesting problem under investigation is that of generalizing the above double bubble proof to $n$ dimensions, hopefully providing a compelling alternative to current proofs for the double bubble in $\mathbb{R}^{n}$. It is hoped that metacalibrations will become a useful tool to solve problems in geometric optimization.

\section{Acknowledgements}

The authors respectfully thank coresearchers James Dilts and Drew Johnson for their assistance and contributions to the proof. This research was funded by the College of Physical and Mathematical Sciences at Brigham Young University.

\section{References}

[Almgren 1976] F. J. Almgren, Jr., "Existence and regularity almost everywhere of solutions to elliptic variational problems with constraints", Mem. Amer. Math. Soc. 4:165 (1976), viii+199. MR 54 \#8420 Zbl 0327.49043

[Foisy et al. 1993] J. Foisy, M. Alfaro, J. Brock, N. Hodges, and J. Zimba, “The standard double soap bubble in $\mathbf{R}^{2}$ uniquely minimizes perimeter", Pacific J. Math. 159:1 (1993), 47-59. MR 94b:53019 Zbl 0738.49023

[Isenberg 1978] C. Isenberg, The science of soap films and soap bubbles, Tieto Ltd., Clevedon, 1978. MR 83b:00001 Zbl 0447.76001

[Milman and Schechtman 1986] V. D. Milman and G. Schechtman, Asymptotic theory of finitedimensional normed spaces, Lecture Notes in Math. 1200, Springer, Berlin, 1986. MR 87m:46038 Zbl 0606.46013

[Morgan 1994] F. Morgan, "Soap bubbles in $\mathbf{R}^{2}$ and in surfaces", Pacific J. Math. 165:2 (1994), 347-361. MR 96a:58064 Zbl 0820.53002

[Siegel 2003] A. Siegel, "A historical review of the isoperimetric theorem in 2-d, and its place in elementary plane geometry", preprint, Courant Institute, 2003, Available at http://www.cs.nyu.edu/ faculty/siegel/SCIAM.pdf.

[Taylor 1976] J. E. Taylor, "The structure of singularities in soap-bubble-like and soap-film-like minimal surfaces”, Ann. of Math. (2) 103:3 (1976), 489-539. MR 55 \#1208a Zbl 0335.49032

[Wichiramala 2004] W. Wichiramala, "Proof of the planar triple bubble conjecture", J. Reine Angew. Math. 567 (2004), 1-49. MR 2005a:53013 Zbl 1078.53010

Received: 2009-10-22 Accepted: 2009-10-23

beccadorff@gmail.com

Mathematics Department, Brigham Young University, Provo, UT 84602, United States

lawlor@mathed.byu.edu Department of Mathematics Education, Brigham Young University, 185 TMCB, Provo, UT 84602, United States

dsampson@byu.net

Mathematics Department, Brigham Young University, Provo, UT 84602, United States http://sites.google.com/site/sampsondcs/

mechanicaltrombone@hotmail.com

Mathematics Department, Duke University, Box 90320 ,

Durham, NC 27708-0320, United States 